\title{
Stability of Multistep Methods for Delay Differential Equations
}

\author{
By Lawrence F. Wiederholt*
}

\begin{abstract}
The absolute and relative stability of linear multistep methods for a finite step size is studied for delay differential equations. The differential equations are assumed linear and the delays a constant integer multiple of the step size. Computable conditions for stability are developed for scalar equations. Plots of the stability regions for several common multistep methods are included. For the integration methods considered, the stability regions for delay differential equations are significantly different from the stability regions for ordinary differential equations.
\end{abstract}

Introduction. The stability of multistep integration methods applied to ordinary differential equations has received considerable attention in the literature [1]-[5], but little has been published on the stability of integration algorithms applied to delay (or retarded) differential equations [6]. An indication of the importance of delay differential equations is evidenced by the many different areas in which they describe physical systems, such as electrostatic charge problems, automatic controls, machine tools, biological systems, and number theory problems. It has been shown that the linear multistep methods used for ordinary differential equations can also be used to generate the solution of delay differential equations, and stability in the limit as the step size tends to zero has been considered [7]-[9]. In this paper the absolute and relative stability of linear multistep methods is considered for delay differential equations when the step size is finite [10]. For several commonly used integration algorithms, plots of the stability region are presented. The delay differential equations will be assumed to be of the form

$$
\frac{d y(t)}{d t}=\sum_{i=1}^{N} f_{i} y\left(t-\omega_{i}\right),
$$

with an initial function $y(t)=\theta(t)$ for $t_{0}-\omega_{i} \leqslant t \leqslant t_{0}$, where $f_{i}$ and $\omega_{i}>0$ are constants, $\omega_{1}=0$ and $y$ is a scalar.

1. Concepts of Stability. The form of the general linear multistep method is given by

$$
u_{n+k}=\sum_{i=0}^{k} a_{i} u_{n+k-i}+h \sum_{i=0}^{k} b_{i} u_{n+k-i}^{\prime},
$$

Received October 31, 1975.

AMS (MOS) subject classifications (1970). Primary 65L99. methods.

Key words and phrases. Delay (or retarded) differential equations, stability of integration

* This paper is based upon the author's Ph. D. dissertation at the University of Wisconsin, June 1970. 
where

$$
\begin{aligned}
k & =\text { degree of multistep method, } \\
a_{i}, b_{i} & =\text { constant coefficients of the method, } \\
h & =\text { step size, } \\
u^{\prime} & =d u / d t, \\
u_{n} & =u(n h)=u(t), \text { which assumes } t_{0}=0, \text { with no loss of generality. }
\end{aligned}
$$

If the exact solution $y_{i}$ is substituted for $u_{i}$ in (1.1), a truncation error at each step $T_{n+k}$ must be added to the right-hand side for the equality to hold. Likewise, if the computed solution $z_{i}$ is substituted for $u_{i}$ in (1.1), a round-off error at each step $\epsilon_{n+k}$ must be added to the right-hand side for the equality to hold. Then the propagation error $e_{n, P}$ is defined as the difference $e_{n, P}=y_{n}-z_{n}$. The notation used is $e_{n, P}$ to denote the general solution and $e_{n}$ to denote only the homogeneous part of the solution.

Stability analysis of a method is a study of the growth of homogeneous solution, $e_{n},\left(\epsilon_{n+k}=0\right.$ and $\left.T_{n+k}=0\right)$ of the propagated error. For stability one is concerned not with the source of the error but only the behavior of the homogeneous solution after some error has been introduced. A numerical integration method is defined as absolutely stable if the homogeneous solution of the propagated error goes to zero as $n$ approaches infinity, i.e. $\lim _{n \rightarrow \infty} e_{n}=0$, and it is defined as relatively stable if the homogeneous solution of the propagated error grows more slowly than the exact solution of the linearized delay equation. Closely connected to the relative and absolute stability of a method is the stability of the delay equation which is being solved. The delay equation (I.1) is defined as stable if for any sufficiently small initial function the solution $y(t)$ approaches zero as $t$ approaches infinity, i.e.,

$$
\lim _{t \rightarrow \infty}|y(t)|=0 \text { for }|y(\tau)|<\delta, \delta>0,-\omega<\tau<0 .
$$

If the solution itself is approaching zero, it is important that the homogeneous solution of the propagated error also approach zero. On the other hand, if the exact solution is unstable, the requirement that the error go to zero is unduly stringent. What is important in this case is that the error grow no faster than the exact solution. Connecting this with the stability of a method, it follows that, in the region of stability of the delay equation, it is necessary to consider the absolute stability of the method, while in the region of instability of the delay equation, it is sufficient to study the relative stability of the method. This is the approach used here. Actually, the relative stability of the method could be considered in both the region of stability and instability of the delay equation; but usually absolute stability is sufficient when the delay equation is stable.

1.1. Absolute Stability of a Method. To study the absolute stability of a method, the usual propagation error equation formed from (1.1) is given as

$$
e_{n+k, P}=\sum_{i=0}^{k} a_{i} e_{n+k-i, P}+h \sum_{i=0}^{k} b_{i} e_{n+k-i, P}^{\prime}+T_{n+k}-\epsilon_{n+k} .
$$

The term $e_{n+k-i}^{\prime}$ of (1.1.1) depends on how $Z_{n+k-i}^{\prime}$ is evaluated. The evaluation of 
$Z_{n+k-i}^{\prime}$ depends on the algorithm and its mode of usage, e.g., the predict, evaluate, correct, evaluate mode; the iterated corrector mode. $e_{n+k-i}^{\prime}$ is determined from (I.1),

$$
e^{\prime}(t)=\sum_{i=1}^{N} f_{i} e\left(t-\omega_{i}\right)
$$

which in discretized form becomes

where

$$
e_{n, P}^{\prime}=\sum_{i=1}^{N} f_{i} e_{n-m_{i}, P}
$$

$$
\begin{array}{cc}
m_{i}=\omega_{i} / h & \text { an integer, } \\
n=t / h & \text { an integer. }
\end{array}
$$

Note that the computation of $Z_{n+k-i}^{\prime}$ is determined by the value of $Z_{n+k-i}$ used in (I.1) which is now reflected in $e_{n-m_{i}, P}$. The homogeneous solution of (1.1.1) is of the familiar form

$$
e_{n}=\sum c_{i} \rho_{i}^{n}
$$

where the $\rho_{i}$ are roots of the characteristic equation.

Assume $T_{n+k}-\epsilon_{n+k}=T$ is constant over a step interval. This leads to the definition that a method is absolutely stable if and only if the magnitude of all roots of its characteristic equation are less than one, $|\rho|<1$. This follows from the arguments used for ordinary differential equations [11], [12]. To illustrate the form of (1.1.1) and its characteristic equation, a predictor-corrector algorithm used in the iterated corrector mode is considered. Equation (1.1.1) becomes for this case

$$
e_{n+k, P}=\sum_{i=0}^{k}\left(a_{i}+b_{i} h f_{1}\right) e_{n+k-i, P}+h \sum_{i=0}^{k} b_{i}\left(\sum_{j=2}^{N} f_{j} e_{n+k-m_{j}-i, P}\right)+T
$$

for which the characteristic equation is

$$
\rho^{M+k}=\sum_{i=0}^{k}\left(a_{i}+b_{i} h f_{1}\right) \rho^{M+k-i}+h \sum_{i=0}^{k} b_{i}\left(\sum_{j=2}^{N} f_{j} \rho^{k-i-m_{j}+M}\right),
$$

where $M=\max _{j=1, \ldots, N} m_{j}$. Other modes are considered in the examples below.

1.2. Relative Stability of a Method. From the definition of relative stability, a computable criterion for relative stability is developed which is based on the comparison of eigenvalues. The approach used is to obtain an approximate solution for both the propagated error equation and the linearized delay equation, and then compare their rate of growth. Starting with the delay equation, one can assume that the delay equation (1.1.2) has a solution of the form

$$
e(t)=\sum_{i=1}^{\infty} c_{i} e^{\lambda_{i} t}
$$

where the $\lambda_{i}$ are roots of the characteristic equation of (1.1.2) given by 


$$
s=f_{1}+\sum_{i=2}^{N} f_{i} e^{-s \omega_{i}}
$$

In discretized form,

$$
e(n h)=\sum_{i=1}^{\infty} c_{i} e^{\lambda_{i} n h}
$$

which, for large $n$, can be approximated by

$$
e(n h) \approx c_{D} e^{\lambda} D^{n h},
$$

where $\lambda_{D}$ is the dominant root which is defined as

$$
\operatorname{Re}\left(\lambda_{D}\right)=\max _{i=1, \ldots, \infty} \operatorname{Re}\left(\lambda_{i}\right) .
$$

Parameterized with respect to $h,(1.2 .1)$ and (1.2.2) become, respectively,

where

$$
\tau=h_{1}+\sum_{i=2}^{N} h_{i} e^{-\tau m_{i}} \quad \text { and } \quad e(n h) \approx c_{D} e^{\tau} D^{n}
$$

$$
\tau=s h, h_{i}=h f_{i} \text { and } \tau_{D}=\lambda_{D} h .
$$

Considering the method, the homogeneous portion of the propagation error equation (1.1.1) has a solution given by (1.1.3) which for large $n$ can be approximated by $e_{n} \approx d_{D} \rho_{D}^{n}$, where $\rho_{D}$ is the dominant root which is defined as

$$
\left|\rho_{D}\right|=\max _{i=1, \ldots, M+k}\left|\rho_{i}\right| .
$$

Given an approximate solution for both the delay equation and the propagation error equation of the method, it is possible to compare their relative rate of growth by comparing $e^{\lambda} D$ and $\rho_{D}$, which will be the dominant growth terms of each solution. For relative stability, the initial conditions are not important, since they only correspond to a translation of the solution which does not affect the rate of growth of the solution.

From the definition of relative stability, it is necessary that the growth of the propagated error be slower than the growth of the exact solution of the linearized delay equation, which implies that the rate of growth of $\rho_{D}$ be less than or equal to the rate of growth of $e^{\tau} D$. In equation form, the condition for relative stability is

$$
\operatorname{Re}\left(\tau_{D}\right) \geqslant \ln \left|\rho_{D}\right|
$$

1.3. Stability of Delay Equation. Before one can proceed further in the stability analysis of a method, it is necessary to know the region of stability of the delay equation in order to define the region where absolute and relative stability are relevant. The region of stability of the delay equation can be determined by studying the behavior of an error introduced in the exact solution [13]. Equation (1.1.2) is the error equation of interest here. The delay equation is stable if and only if the error equation is stable. It is well known that (1.1.2) is stable if and only if $\operatorname{Re}(s) \leqslant 0$ for all roots of its characteristic equation (1.2.1). To determine the boundary between the stable and unstable regions, set $s=j b$, from which (1.2.1) becomes 


$$
0=f_{1}+\sum_{i=2}^{N} f_{i} \cos \omega_{i} b, \quad b=-\sum_{i=2}^{N} f_{i} \sin \omega_{i} b .
$$

2. Stability Region for Several Integration Algorithms. In this section the regions of stability for a nonzero step size are determined for several integration algorithms when applied to delay differential equations. The case treated here is that of a single delay for which (I.1) becomes

$$
y^{\prime}(t)=f_{1} y(t)+f_{2} y\left(t-\omega_{2}\right) .
$$

For this case Eq. (1.3.1) for the stability region of the delay equation becomes

$$
f_{2}=-b / \sin \omega b, \quad f_{1}=b \operatorname{cotan} \omega b,
$$

which is plotted in Fig. 2.1. This determines the region in the $f_{1}-f_{2}$ plane in which absolute stability of the method is relevant.

The first method chosen was the familiar Euler's method given by $y_{n+1}=y_{n}+$ $h f_{n}$, which has the characteristic equation $\rho^{m+1}-\left(1+h_{1}\right) \rho^{m}-h_{2}=0$.

The region of absolute and relative stability for Euler's method is defined in Fig. 2.2 where $h_{i}=h f_{i}$. Figure 2.1, parameterized with respect to $h$ and evaluated at $m=$ 2, is superimposed on Fig. 2.2. Two values of $m$, an even and an odd, were used to present some typical results. Other values of $m$ result in modification of these regions.

Milne's predictor-corrector algorithm was the second method considered, which is given by

$$
\begin{aligned}
& y_{n+1}^{P}=y_{n-3}+(4 h / 3)\left(2 y_{n}^{\prime}-y_{n-1}^{\prime}+2 y_{n-2}^{\prime}\right), \\
& y_{n+1}=y_{n-1}+(h / 3)\left(y_{n+1}^{P^{\prime}}+4 y_{n}^{\prime}+y_{n-1}^{\prime}\right) .
\end{aligned}
$$

Since in practice the predict-correct mode is commonly used, it is the only mode considered here. The characteristic equation is given by

$$
\begin{aligned}
\rho^{2 m+3}= & \left(1-\frac{13 h_{1}}{9}\right) \rho^{2 m+1} \\
+\frac{1}{3}\left[\rho^{2 m+2}\left(4 h_{1}+\frac{8 h_{1}^{2}}{3}\right)+\rho^{2 m}\left(\frac{8 h_{1}^{2}}{3}\right)\right. & \\
& +\rho^{2 m-1} h_{1}+\rho^{m+2}\left(4 h_{2}+\frac{16 h_{1} h_{2}}{3}\right) \\
& +\rho^{m+1}\left(h_{2}-\frac{8 h_{1} h_{2}}{3}\right)+\rho^{m}\left(\frac{16 h_{1} h_{2}}{3}\right)+h_{2} \rho^{m-1} \\
& \left.\quad+\frac{8}{3} h_{2}^{2} \rho^{2}-\frac{4 h_{2}^{2}}{3} \rho+\frac{8}{3} h_{2}^{2}\right] .
\end{aligned}
$$

The regions of absolute and relative stability are plotted in Figs. 2.3 and 2.4, respectively, as well as the parameterized stability curve for the delay equation.

The Adams-Moulton predictor-corrector algorithm was the third method chosen which is given by

$$
\begin{aligned}
& y_{n+1}^{P}=y_{n}+(h / 24)\left(55 y_{n}^{\prime}-59 y_{n-1}^{\prime}+37 y_{n-2}^{\prime}-9 y_{n-3}^{\prime}\right), \\
& y_{n+1}=y_{n}+(h / 24)\left(9 y_{n+1}^{P^{\prime}}+19 y_{n}^{\prime}-5 y_{n-1}^{\prime}+y_{n-2}^{\prime}\right) .
\end{aligned}
$$


In the predict-correct mode the characteristic equation is given by

$$
\begin{aligned}
& \rho^{2 m+4}=\rho^{2 m+3}\left(1+\frac{5 h_{1}}{6}+\frac{55 h_{1}^{2}}{(24)^{2}}\right) \\
&+\frac{1}{24}\left[-\rho^{2 m+2}\left(\frac{59}{24} h_{1}^{2}+5 h_{1}\right)+\rho^{2 m+1}\left(\frac{37 h_{1}^{2}}{24}+h_{1}\right)-\frac{3}{8} \rho^{2 m} h_{1}^{2}\right. \\
&+\rho^{m+3}\left(\frac{55}{12} h_{1} h_{2}+20 h_{2}\right)-\rho^{m+2}\left(\frac{59}{12} h_{1} h_{2}+5 h_{2}\right) \\
&+\rho^{m+1}\left(\frac{37}{12} h_{1} h_{2}+h_{2}\right)-\rho^{m}\left(\frac{3}{4} h_{1} h_{2}\right) \\
&\left.+\frac{55}{24} \rho^{3} h_{2}^{2}-\frac{59}{24} \rho^{2} h_{2}^{2}+\frac{37}{24} \rho h_{2}^{2}-\frac{3}{8} h_{2}^{2}\right] .
\end{aligned}
$$

The regions of absolute and relative stability are plotted in Figs. 2.5 and 2.6, respectively, as well as the stability curve for the delay equation.

For the methods considered, it is of interest to compare the regions of absolute and relative stability for delay differential equations and ordinary differential equations. Note that for $h_{2}=0$, the absolute stability analysis of a delay differential equation reduces to that of an ordinary differential equation. The result is that the plots of the regions of absolute stability for delay differential equations also include the region of absolute stability for ordinary differential equations. The absolute stability region for ordinary differential equations is just the absolute stability interval on the $h_{1}$-axis for delay differential equations. For each integration method considered, the region of relative stability for ordinary differential equations is the entire positive $h_{1}$-axis. Comparing the stability regions for ordinary differential equations and delay differential equations, one can see that the stability regions are restricted for delay differential equations.

Conclusion. The concepts of absolute and relative stability of linear multistep methods for a finite step size have been extended to delay differential equations. The change in the stability region as one moves away from the $h_{1}$-axis in the plots of the stability region emphasizes the importance of accounting for the delay terms when considering the stability of an integration algorithm for solving delay differential equations.

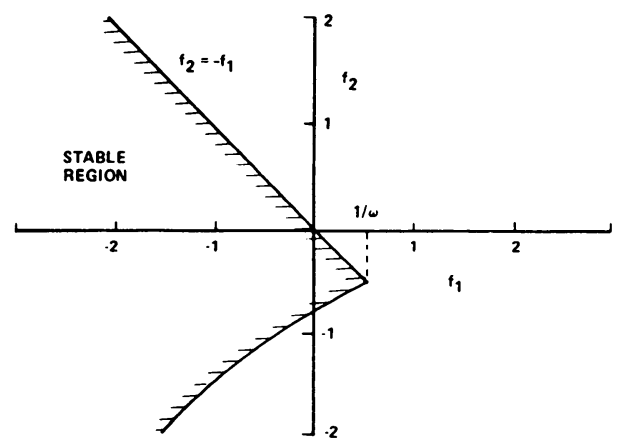

FIGURE 2.1. Stability region for the delay differential equation. 


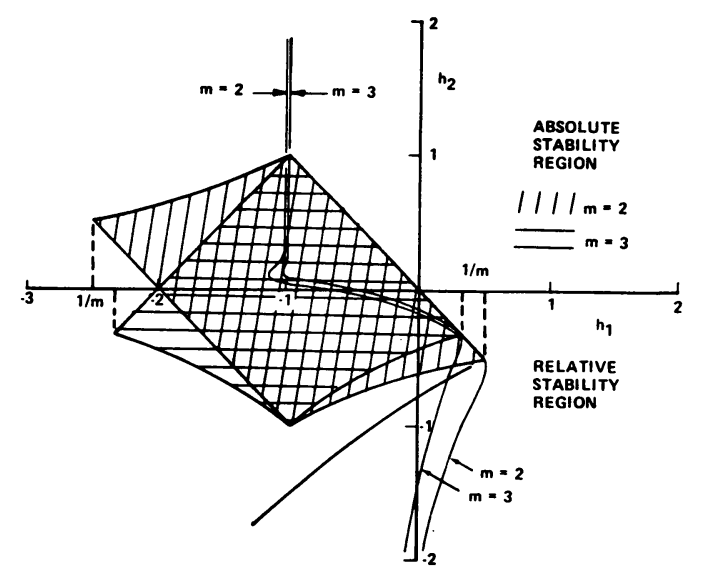

FIGURE 2.2. Absolute and relative stability regions for Euler's method.

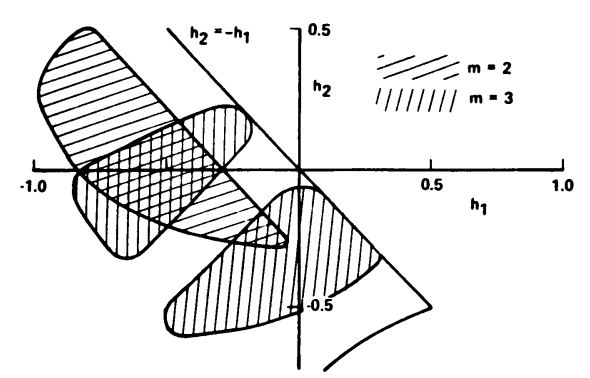

FIGURE 2.3. Absolute stability region for Milne's method.

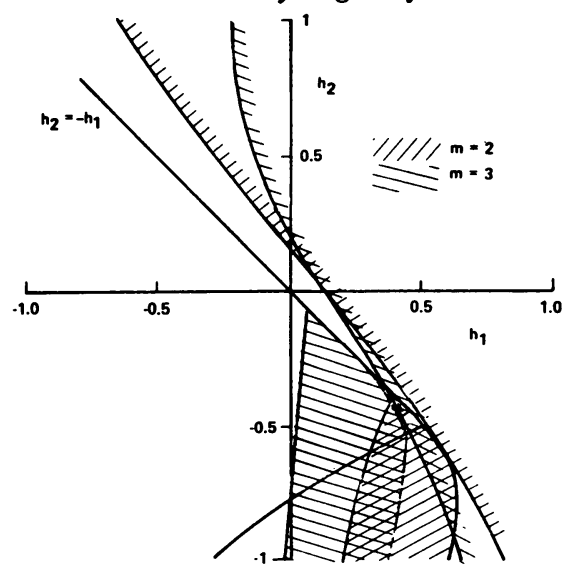

FIGURE 2.4. Relative stability region for Milne's method.

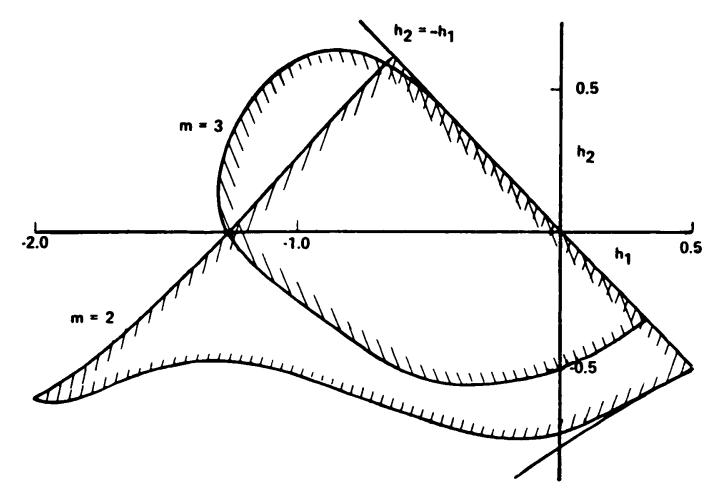

FIGURE 2.5. Absolute stability region for the Adams-Moulton method. 


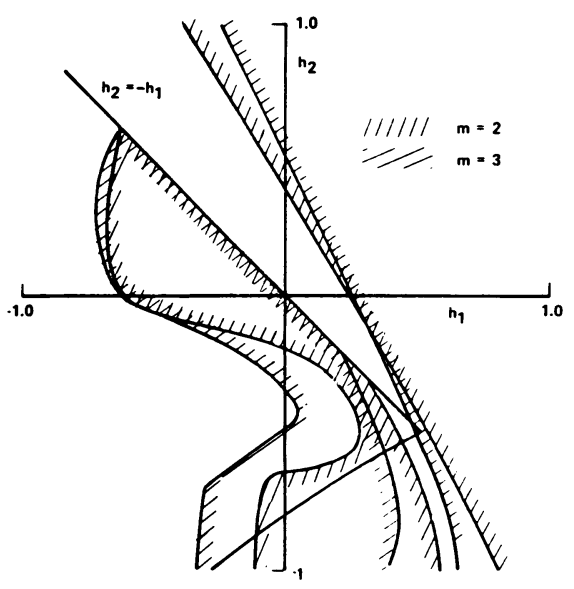

FIGURE 2.6. Relative stability region for the Adams-Moulton method.

Calspan Corporation

P. O. Box 235

Buffalo, New York 14221

1. P. E. CHASE, "Stability properties of predictor-corrector methods for ordinary differential equations," J. Assoc. Comput. Mach., v. 9, 1962, pp. 457-468. MR 29 \#738.

2. R. R. BROWN, J. D. RILEY \& M. M. BENNETT, "Stability properties of Adams-Moulton type methods," Math. Comp., v. 19, 1965, pp. 90-96. MR 31 \#2829.

3. A. RALSTON, "Relative stability in the numerical solution of ordinary differential equations," SIAM Rev., v. 7, 1965, pp. 114-125. MR 31 \#2831.

4. R. W. HAMMING, "Stable predictor-corrector methods for ordinary differential equations," J. Assoc. Comput. Mach., v. 16, 1959, pp. 37-47. MR 21 \#973.

5. W. B. GRAGG \& H. J. STETTER, "Generalized multistep predictor-corrector methods," J. Assoc. Comput. Mach., v. 11, 1964, pp. 188-209. MR 28 \#680.

6. R. K. BRAYTON \& R. A. WILLOUGHBY, "On the numerical integration of a symmetric system of difference-differential equations of neutral type," J. Math. Anal. Appl., v. 18, 1967, pp. 182-189. MR 35 \#3926.

7. L. TAVERNINI, Numerical Methods for Volterra Functional Differential Equations, Ph. D. Thesis, University of Wisconsin, 1969.

8. M. A. FELDSTEIN, Discretization Methods for Retarded Ordinary Differential Equations, $\mathrm{Ph}$. D. Thesis, University of California, Los Angeles, 1964.

9. M. N. SPYKER, Stability and Convergence of Finite-Difference Methods, Ph. D. Thesis, Centraal-Reken-Instituul, Ryksuniversiteil, Leiden, The Netherlands, 1969.

10. L. F. WIEDERHOLT, Numerical Integration of Delay Differential Equations, Ph. D. Thesis, University of Wisconsin, 1970.

11. P. HENRICI, Discrete Variable Methods in Ordinary Differential Equations, Wiley, New York, 1962, p. 218. MR 24 \#B1772.

12. R. L. CRANE \& R. J. LAMBERT, "Stability of a generalized corrector formula," J. Assoc. Comput. Mach., v. 9, 1962, pp. 104-117. MR 24 \#B1283.

13. A. HALANAY, Differential Equations: Stability, Oscillations, Time Lags, Academic Press, New York, 1966, p. 349. MR 35 \#6938. 